\title{
Assessment of Sperm Viability by Measurement of ATP, Membrane Integrity and Motility in Frozen/Thawed Bull Semen
}

Control of sperm quality after commercial freezing/thawing of bull semen is still restricted to the subjective assessment of sperm motility, despite its low correlation to fertility (Söderquist et al. 1991, Kjaestad et al. 1993). Although no single in vitro method has yet been designed to predict the fertilizing ability of a given semen sample, the quantitation of viable spermatozoa (with intact plasma and acrosome membranes, and metabolically active) seems to be most promising (Woelders et al. 1991). The present report describes the use of a bioluminiscence technique to determine ATP-levels and a novel supravital stain (using fluorescent dyes) to assess the amount of viable spermatozoa in frozen/thawed semen from 3 A.I. dairy bulls with significantly different motility after thawing.

Frozen/thawed semen from 3 7-year-old bulls of the Swedish Red and White breed was used. Straws $\left(0.25 \mathrm{ml}, 20 \times 10^{6}\right.$ spermatozoa $)$ from a single freezing operation/bull (10 replicates), were thawed by immersion in water at $35^{\circ} \mathrm{C}$ for $12 \mathrm{sec}$ and immediately evaluated. Sperm motility was estimated on a microscope equipped with phase contrast optics and a thermal plate at $37^{\circ} \mathrm{C}(450 \times)$. ATP determinations were done using a bioluminiscence technique with the Firezyme Sperm Viability Test $^{\circledR}$ System $^{\circledR}$
(SVT, Firezyme Diagnostics Inc, Halifax, Canada), in which 2 aliquots $(50 \mu \mathrm{l})$ from each straw were tested, one aliquote to determine (after disruption of the cells) the total amount of ATP (T-ATP), and the second to quantify the amount of free ATP outside competent cells in the seminal fluid (blank, EC-ATP). LuciferinLuciferase was used as ATP-monitoring agent, and the light reaction was measured into a luminometer chamber (SVT 2.5 Bioluminometer ${ }^{\mathrm{R}}$, Minitüb GmbH, Landshut, Germany) at $20^{\circ} \mathrm{C}$. Preparation of reagents (Luciferin-Luciferase solution, buffers), internal calibration and testing procedures were performed as indicated by the manufacturers. The data of the light output was later integrated and converted into relative light units, the reading used to determine sample ATP from the computer-generated standard curve. The results of ATP were expressed as total and extracellular ATP, in $\mu \mathrm{g} / \mathrm{ml}$. Net ATP within competent cells, i.e. the difference between T-ATP and EC-ATP, was used to calculate the concentration of viable spermatozoa $/ \mathrm{ml}$ of semen and finally, per straw (as VSS, $10^{6} /$ straw).

Sperm membrane integrity was assessed by incubating the frozen/thawed spermatozoa in a staining medium containing $4 \mu \mathrm{l}$ of the membrane-impermeant DNA-marker Ethidium ho- 


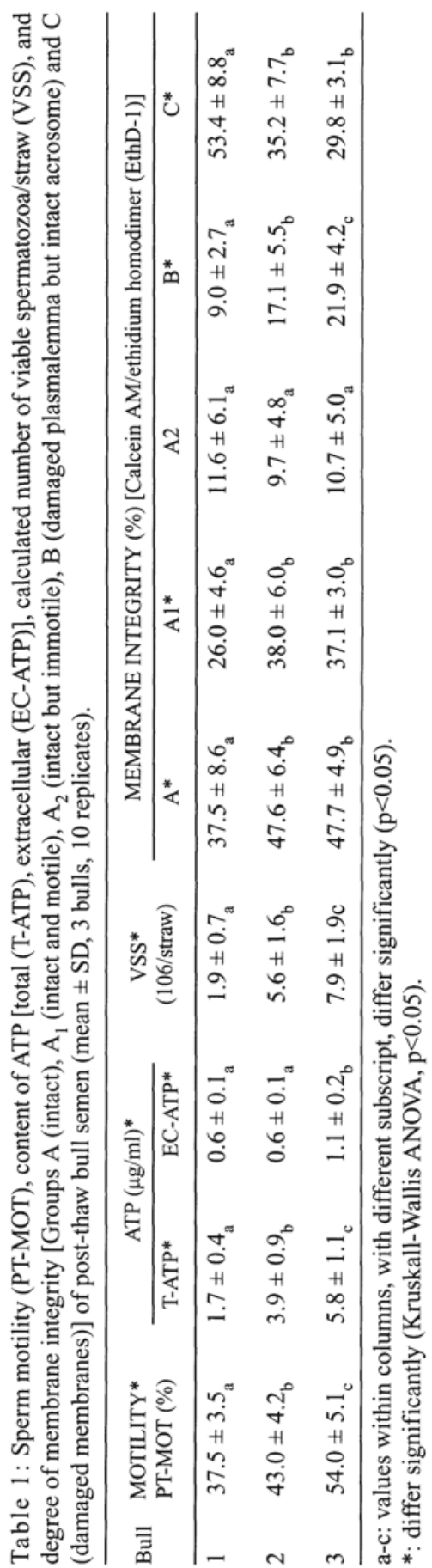

modimer (EthD-1) (Molecular Probes Inc, Eugene OR, USA) and $10 \mu \mathrm{l}$ of the membranepermeable cytoplasmic esterase-marker Calcein AM (Molecular Probes Inc) in $1 \mathrm{ml}$ of Phosphate Buffer Solution (PBS). The reagents are previously prepared at a concentration of 1 $\mathrm{mg} / \mathrm{ml}$ in DMSO and kept frozen as stock solutions. The staining medium was prepared not more than $1 \mathrm{~h}$ before use and kept dark. Semen $(20 \mu \mathrm{l})$ was added to $20 \mu \mathrm{l}$ of staining medium and incubated in darkness for 15-30 min at 30$35^{\circ} \mathrm{C}$. Subsamples $(5 \mu \mathrm{l})$ of the stained suspension were placed on clean microscope slides and overlaid carefully with coverslips. Random fields were observed under $400 x$ magnification with epifluorescence UV-illumination on a Diaplan Leitz microscope. Staining (Calcein AM: green, EthD: red) was assessed using a H3-I3 filter block (Leitz 513673-719, blue excitation range, with a BP420-490 excitation filter, a RKP510 dichromatic mirror and a LP520 suppressor filter). For quantitation of sperm membrane integrity, at least 200 cells were counted in each of two $5 \mu \mathrm{l}$ aliquots from a stained sample. The counted sperm cells were classified as A: having an intact plasmalemma when green with Calcein and unstained with EthD (they were divided into category $A_{1}$ when motile and $\mathrm{A}_{2}$ if immotile); $\mathrm{B}$ : having a damaged plasmalemma but an intact acrosome, when the acrosome stained green with Calcein but the postacrosomal region stained red with $\mathrm{EthD}$ and; $\mathrm{C}$ : having both a damaged plasmalemma and acrosomal membrane when the cells were unstained with Calcein but stained red with EthD.

Sperm concentration was confirmed by counting thawed semen samples (3 replicates) in a Bürker chamber. The morphology of 500 spermatozoa/bull were counted on dry smears stained with carbol-fuchsine on a light microscope $(1000 \times)$. The morphology of the tail, mid-piece and acrosomes was evaluated after counting 200 spermatozoa in wet smears (fixed 
in buffered formol-saline) with a phase-contrast microscope $(1000 \times)$. Dry smears were also checked for others cells than spermatozoa after staining with Papanicolau's stain (Ortho Diagnostics, New Jersey, USA). Statistical analysis (Comparison of means, Kruskal-Wallis ANOVA, Correlations) of the results obtained were performed using the CSS:STATISTICA $^{\mathrm{R}}$ programme software (STATISTICA for Windows, Statsoft, Tulsa OK, USA, 1994).

The sperm morphology appeared within normal limits for the age of the bulls, with a frequency of sperm head shape abnormalities of $13.0 \pm 4.6 \%, 3.6 \pm 1.7 \%$ of immature spermatozoa (proximal droplets), $7.0 \pm 4.7 \%$ of abnormal acrosomes and $3.7 \pm 1.3 \%$ of abnormal tail shapes. The frequencies of sperm abnormalities differed significantly among bulls $(\mathrm{p}<0.05)$. The data for motility, ATP-levels and membrane integrity are presented in Table 1 as means \pm SD. The overall post-thaw motility (PT-MOT) of the assayed bulls was $45 \pm 8.1 \%$, differing significantly among bulls $(\mathrm{P}<0.001)$, with a mean concentration of $20.8 \pm 1.2$ million sperm/straw (n.s.). The amount of T-ATP varied significantly $(\mathrm{p}<0.001)$ among the bulls, being positively correlated with the PT-MOT $(r=$ $0.81, \mathrm{p}<0.05$ ), while the amount of extracellular ATP showed less variation among the animals tested. The amount of spermatozoa with intact plasmalemma, as detected with the supravital markers (Group A), also varied significantly between bulls, but not between those with a post-thaw motility above $40 \%$ (n.s.). A similar tendency was recorded between the subjective evaluation of motility and the percentage of cells having intact membranes which also had progressive motility $\left(\mathrm{A}_{1}\right)$. The amounts of sperm cells with damaged plasma and acrosome membranes (Group C) showed a clear, and significant inverse tendency $(\mathrm{p}<0.05)$. The SVT (ATP-based) system calculated mean figures of $25 \%$ of viable spermato- zoa/straw (VSS, mean ranges $=10-38 \%$ ) while the supravital markers identified $33 \%$ of spermatozoa with intact plasmalemma and progressive motility.

The ATP-based calculation of viable spermatozoa per straw (VSS) correlated positively with PT-MOT $(r=0.79, p<0.01)$. Both methods for calculation of viable spermatozoa/straw (ATP and supravital stain, $\mathrm{A}_{1}$ group, i.e. those producing and consuming ATP) were also correlated $(\mathrm{r}=0.58, \mathrm{P}<0.05)$. The ATP-method validated as well when comparing the amount of EC-ATP and the amount of spermatozoa with damaged plasmalemma $[\mathrm{r}=-0.49$ (B) to 0.55 (C), $\mathrm{p}<0.05$ ], i.e. leaking, and thus stained with the DNA-stain EthD-1. From these preliminary results it can be concluded that both the SVTmethod (based on ATP determination) and/or the supravital loading with Calcein AM (marker for membrane integrity) can be used to evaluate the amount of viable bull spermatozoa after freezing/thawing. It remains however, to determine which correlation do these methods have with the fertility of the in vitro assayed samples.

\section{Acknowledgements}

The authors are indebted to Annika Rikberg and Karin Selin-Wretling for technical assistance. Dr. Januskauskas is holder of a scholarship from the Royal Academy of Forestry and Agriculture (KSLA). This work received financial support from the Stiftelsen Lantbruksforskning, Sweden.

\section{A. Januskauskas, and H. Rodriguez-Martinez}

Department of Obstetrics \& Gynaecology, Faculty of Veterinary Medicine, Swedish University of Agricultural Sciences, P.O. Box 7039, Uppsala, Sweden.

\section{References}

Kjaestad H, Ropstad E. \& Andersen Berg K.: Evaluation of spermatological parameters used to predict the fertility of frozen bull semen. Acta vet scand, 1993, 34, 299-303.

Söderquist L, Rodriguez-Martinez H, and Jansson L: Post-thaw motility, ATP content and cytochrome 
C oxidase activity of A.I. bull spermatozoa in relation to fertility. J. vet. Med. A, 1991, 38, 165174.
Woelders $H$ : Overview of in vitro methods for evaluation of semen quality. Reprod. Dom. Anim., 1991, Suppl 1 (Johnson, L.A. \& Rath, D.), pp. 145-164.

(Received February 7, 1995; accepted August 23, 1995).

Reprints may be obtained from: H. Rodriquez-Martinez, Department of Obstetrics \& Gynaecology, Faculty of Veterinary Medicine, Swedish University of Agricultural Sciences, P.O. Box 7039 Uppsala, Sweden. 